\title{
PLANAR GRAPHS WITHOUT SHORT EVEN CYCLES ARE NEAR-BIPARTITE
}

\author{
RUNRUN LIU ${ }^{1,2}$ GEXIN YU²,3 \\ ${ }^{1}$ Department of Mathematics, Zhejiang Normal University, Jinhua, 321004, China. \\ ${ }^{2}$ Department of Mathematics and Statistics, Central China Normal University, Wuhan, 430079, China. \\ ${ }^{3}$ Department of Mathematics, William 83 Mary, Williamsburg, VA, 23185, USA.
}

\begin{abstract}
A graph is near-bipartite if its vertex set can be partitioned into an independent set and a set that induces a forest. It is clear that near-bipartite graphs are 3-colorable. In this note, we show that planar graphs without cycles of lengths in $\{4,6,8\}$ are near-bipartite.
\end{abstract}

\section{INTRODUCTION}

A graph is $k$-degenerate if each subgraph has a vertex of degree at most $k$. Every $k$-degenerate graph is $(k+1)$-colorable. Borodin [1] in 1976 suggested to partition the vertex set of a graph into two sets that induce graphs with better degeneracy properties. A graph $G$ is $(a, b)$-partitionable if its vertices can be partitioned into sets $A$ and $B$ such that the subgraph induced by $A$ is $a$ degenerate and the subgraph induced by $B$ is $b$-degenerate. Borodin [1 conjectured that every planar graph, which is 5-degenerate, is (1,2)- and (0,3)-partitionable. Thomassen [10, 11] confirmed these conjectures.

Clearly a graph is bipartite if and only if it is $(0,0)$-partitionable. A graph is called near-bipartite if it is $(0,1)$-partitionable. Recognizing near-bipartite graphs can be seen as restricted variants of the 3-coloring problem, which is well known to be NP-complete [9]. Borodin and Glebov [2] showed that every planar graph of girth at least 5 is near-bipartite (see [7] for an extension of this result). Dross, Montassier, and Pinlou [6] asked whether every triangle-free planar graph is near-bipartite. Borodin, Glebov, Raspaud, and Salavatipour [5] proved that planar graphs without cycles of length from 4 to 7 are 3 -colorable. Wang and Chen [12] showed that planar graphs without $\{4,6,8\}$-cycles are 3-colorable. In this paper, we improved this result by showing that planar graphs without $\{4,6,8\}$-cycles are near-bipartite. Some tricks in the proof resemble those first appeared in [2, 5] and further developed in [3, 4.

Theorem 1.1. Every planar graph without $\{4,6,8\}$-cycles is near-bipartite.

An IF-coloring of a graph is a partition of its vertices into two parts such that one part colored $I$ induces an independent set and the other part colored $F$ induces a forest. Given a graph $G$ and a cycle $C$ in $G$, an $I F$-coloring $\phi_{C}$ of $G[V(C)]$ superextends to $G$ if there exists an $I F$-coloring $\phi_{G}$ of $G$ that extends $\phi$ with the property that there is no path joining two vertices of $C$ all of whose vertices are colored $F$ and do not belong to $C$. We say that $C$ is superextendable to $G$ if every

E-mail address: gyu@wm.edu.

The research was partially supported by NSFC (11728102) and the NSA grant H98230-16-1-0316. 
$I F$-coloring $\phi_{C}$ of $G[V(C)]$ superextends to $G$. When we wish to specify $G$, we will say $(G, C)$ is superextendable.

Instead of Theorem 1.1, we actually proved a stronger result:

Theorem 1.2. For every planar graph $G$ without $\{4,6,8\}$-cycles and any cycle $C$ in $G$ of length at most 12, $(G, C)$ is superextendable.

By [2], every planar graph of girth 5 has an $I F$-coloring. Since $G$ contains no 4-cycles, $G$ must have a triangle. Thus Theorem 1.1 follows from Theorem 1.2 easily.

Graphs mentioned in this paper are all simple. Let $d(v)$ be the degree of $v$. A $k$-vertex $\left(k^{+}\right.$ vertex, $k^{-}$-vertex) is a vertex of degree $k$ (at least $k$, at most $k$ ). The same notation will be applied to faces and cycles. Let $C$ be a cycle of a plane graph $G$. We use $\operatorname{int}(C)$ and $\operatorname{ext}(C)$ to denote the sets of vertices located inside and outside $C$, respectively. The cycle $C$ is called a separating cycle if $\operatorname{int}(C) \neq \emptyset \neq \operatorname{ext}(C)$. We still use $C$ to denote the set of vertices of $C$. An $F$-cycle is a cycle whose vertices are all colored $F$, and an $F$-path is path whose vertices are colored $F$.

\section{Reducible Configurations}

Let $\left(G, C_{0}\right)$ be a counterexample to Theorem 1.2 with minimum $\sigma(G)=|V(G)|+|E(G)|$, where $C_{0}$ is a cycle of length at most 12 in $G$ that is precolored. If $C_{0}$ is a separating cycle, then $C_{0}$ is superextendable in both $G-\operatorname{ext}\left(C_{0}\right)$ and $G-\operatorname{int}\left(C_{0}\right)$. Thus, $C_{0}$ is superextendable in $G$, contrary to the choice of $C_{0}$. Thus, we may assume that $C_{0}$ is the boundary of the outer face of $G$ in the rest of this paper. Call a vertex $v$ internal if $v \notin C_{0}$, call a face $f$ internal if $f \neq C_{0}$ and truly internal if $V(f) \cap V\left(C_{0}\right)=\emptyset$.

Lemma 2.1. Every internal vertex in $G$ has degree at least 3.

Proof. Suppose there is an internal $2^{-}$-vertex $v$ in G. By minimality of $\left(G, C_{0}\right)$, the $I F$-coloring of $C$ can superextend to $G-v$. If $v$ has a neighbor colored $I$, then color $v$ with $F$, otherwise, color $v$ with $I$. In all cases, $C_{0}$ superextends to $G$, a contradiction.

Lemma 2.2. The graph $G$ has no separating cycle of length at most 12.

Proof. Suppose otherwise that $C$ is a separating cycle of length at most 12 in $G$. Then $C$ is inside of $C_{0}$. By the minimality of $\left(G, C_{0}\right),\left(G-\operatorname{int}(C), C_{0}\right)$ is superextendable, and after that, $G[V(C)]$ is colored. By the minimality of $\left(G, C_{0}\right)$ again, $(C \cup \operatorname{int}(C), C)$ is superextendable. Thus, $\left(G, C_{0}\right)$ is superextendable, a contradiction.

Lemma 2.3. The following are true about $C_{0}$.

(1) $C_{0}$ is chordless.

(2) Every non-adjacent pair $v_{0}$ and $v_{1}$ on $C_{0}$ have no internal common neighbors.

Proof. (1) If $C_{0}$ has a chord, then by Lemma 2.2, $V\left(C_{0}\right)=V(G)$. But by definition, the precoloring of $C_{0}$ is already a good IF-coloring of $G$.

(2) Let $w$ be an internal common neighbor of $v_{0}, v_{1} \in V\left(C_{0}\right)$ with $v_{0} v_{1} \notin E(G)$. Let $P_{1}$ and $P_{2}$ be the two paths of $C_{0}$ with ends $v_{0}$ and $v_{1}$. Then both $P_{1}+v_{0} w v_{1}$ and $P_{2}+v_{0} w v_{1}$ are cycles of length at most 12. By Lemma 2.2, $N(w) \subset V\left(C_{0}\right)$. Note that $d(w) \geq 3$ by Lemma 2.1. So $w$ has at least one neighbor, by symmetry say $v_{2}$ is on the segment of $C_{0}$ from $v_{1}$ to $v_{0}$ in the clockwise order. Let $P_{i}^{\prime}$ be the path of $C_{0}$ with ends $v_{i}, v_{i+1}$ for $i \in$ [3] (index module 3 ) in the clockwise order. Let $C_{i}=P_{i}^{\prime}+v_{i} w v_{i+1}$ for $i \in[3]$. Note that $\left|C_{1}\right|+\left|C_{2}\right|+\left|C_{3}\right| \leq 18$. Without loss of generality, let 
$\left|C_{1}\right| \leq\left|C_{2}\right| \leq\left|C_{3}\right|$. Since $G$ contains no $\{4,6,8\}$-cycles, $\left|C_{1}\right| \in\{3,5\}$; and when $\left|C_{1}\right|=3,\left|C_{2}\right| \geq 9$; when $\left|C_{1}\right|=5,\left|C_{2}\right| \geq 7$. In any case, $\min \{3+9+9,5+7+7\}>18$, a contradiction.

Lemma 2.4. Every truly internal 5-face is incident to at most four 3-vertices.

Proof. Suppose otherwise that $f=\left[v_{1} v_{2} v_{3} v_{4} v_{5}\right]$ is a truly internal 5 -face with five 3 -vertices. Let $u_{i}$ be the neighbor of $v_{i}$ not on $f$ for $i \in[5]$. Let $G^{\prime}$ be the graph obtained by identifying $v_{3}$ and $u_{1}$ of $G-\left\{v_{1}, v_{2}, v_{4}, v_{5}\right\}$. Note that if the identification creates an edge between vertices of $C_{0}$, then $u_{1}, u_{3} \in V\left(C_{0}\right)$, but the path between them along $C_{0}$ with $u_{1} v_{1} v_{2} v_{3} u_{3}$ forms a separating cycle of length at most 12, a contradiction to Lemma 2.2. It follows that the precoloring of $C_{0}$ remains valid in $G^{\prime}$. Furthermore, if there is a path $Q$ of length at most 8 between $v_{3}$ and $u_{1}$, then $G$ has a separating cycle of length at most 11 which is obtained from the path $u_{1} v_{1} v_{2} v_{3}$ and $Q$, contrary to Lemma 2.2. So $G^{\prime}$ contains no $\{4,6,8\}$-cycles. Since $\left|V\left(G^{\prime}\right)\right|<|V(G)|$, we can extend the coloring of $C_{0}$ to $G^{\prime}$. Now we can extend this coloring to an $I F$-coloring of $G$ in the following way.

First assume that the new vertex created by the identification is colored $I$. Then we color $u_{1}$ and $v_{3}$ with $I$ and $v_{1}, v_{2}, v_{4}$ with $F$. If $u_{5}$ is colored with $F$, then color $v_{5}$ with $I$. If $u_{5}$ is colored with $I$, then color $v_{5}$ with $F$, which is invalid only if $u_{2}$ and $u_{4}$ are both colored $F$, in which case, we recolor $v_{3}$ with $F$ and $v_{2}, v_{4}$ with $I$. Note that we create neither $F$-cycle nor $F$-path between two vertices of $C_{0}$.

Now assume that the new vertex created by the identification is colored $F$. First we color $u_{1}$ and $v_{3}$ with $F$. Note that there exists no $F$-path between $u_{1}$ and $v_{3}$ in $G$. If $u_{4}$ is colored $I$, then color $v_{1}, v_{4}$ with $F$, and for each $i \in\{2,5\}$, color $v_{i}$ with a color different from $u_{i}$; this is not a valid coloring only if $u_{2}$ and $u_{5}$ are both colored $I$, in which case we recolor $v_{1}$ with $I$. So we may assume that $u_{4}$ is colored $F$. Color $v_{1}, v_{4}$ with $I$ and $v_{2}, v_{3}, v_{5}$ with $F$, we obtain a valid coloring, unless we create an $F$-cycle or an $F$-path between two vertices of $C_{0}$. In the bad cases, $u_{2}$ and $u_{3}$ are colored $F$. We may further assume that $u_{5}$ is colored $F$, for otherwise we can color $v_{2}, v_{4}$ with $I$ and $v_{1}, v_{3}, v_{5}$ with $F$ to obtain a valid coloring.

Consider the case that $u_{i}$ is colored $F$ for $i \in[5]$. Note that there is no $F$-path between $u_{1}$ and $u_{3}$, and there cannot exist $F$-path between $u_{1}$ and $C_{0}$ and between $u_{3}$ and $C_{0}$ at the same time, since $G^{\prime}$ has a valid coloring. By symmetry, we may assume that there is no $F$-path between $u_{3}$ and $C_{0}$. There must be an $F$-path, say $P$, between $u_{2}$ and $u_{3}$, for otherwise we obtain a valid coloring by coloring $v_{2}, v_{3}, v_{5}$ with $F$ and $v_{1}, v_{4}$ with $I$. We color $v_{1}, v_{2}, v_{4}$ with $F$ and $v_{3}, v_{5}$ with $I$. Since the $F$-path $u_{1} v_{1} v_{2} u_{2}$ in $G$ can be replaced with the $F$-path $u_{1}\left(v_{3}\right) u_{3} P u_{2}$ in $G^{\prime}$, this cannot be $F$-cycle or $F$-path containing $u_{1} v_{1} v_{2} u_{2}$ in $G$. Therefore we obtain a valid coloring.

A 3-vertex $v \notin C_{0}$ is bad if $v$ is on a 3-face. A tetrad in a plane graph is a path $v_{1} v_{2} v_{3} v_{4}$ of four internal 3 -vertices contained in the boundary of a face, so that both $v_{1} v_{2}$ and $v_{3} v_{4}$ are edges of triangles.

Lemma 2.5. G contains no tetrad. Consequently, no face of $G$ is incident with five consecutive bad vertices. Furthermore, if a face of $G$ is incident with consecutive vertices $v_{0}, v_{1}, \ldots, v_{5}$ and the vertices $v_{1}, \ldots, v_{4}$ are bad, then the edges $v_{0} v_{1}, v_{2} v_{3}$ and $v_{4} v_{5}$ are incident with triangles.

Proof. Let $v_{1} v_{2} v_{3} v_{4}$ be a tetrad in $G$. Let $N\left(v_{1}\right)=\left\{x, v_{2}, v_{1}^{\prime}\right\}$ and $N\left(v_{4}\right)=\left\{y, v_{3}, v_{4}^{\prime}\right\}$, where $x, y$ are not in the triangles. Let $G^{\prime}$ be the graph obtained by identifying $y$ and $v_{1}^{\prime}$ of $G-\left\{v_{1}, v_{2}, v_{3}, v_{4}\right\}$. Note that the identification does not create a chord in $C_{0}$ or identify two vertices of $C_{0}$. For otherwise, there exists a path $P$ of length 4 or 5 , which is internally disjoint from $C_{0}$, between two 
vertices of $C_{0}$, and since $\left|C_{0}\right| \leq 12$, there exists a cycle in $P \cup C_{0}$ of length at most 11 that separates $x$ and $v_{4}$, contrary to Lemma 2.2. There is no path $Q$ of length at most 8 between $y$ and $v_{1}^{\prime}$, for otherwise $G$ would have a cycle separating $K$ of length at most 12 that is obtained from the path $y v_{3} v_{2} v_{1} v_{1}^{\prime}$ and $Q$, contrary to Lemma 2.2. Thus, no new $8^{-}$-cycles are created in $G^{\prime}$. This implies that $G^{\prime}$ contains no $\{4,6,8\}$-cycles. Since $\left|V\left(G^{\prime}\right)\right|<|V(G)|$, the precoloring of $C_{0}$ can be extended to an $I F$-coloring of $G^{\prime}$. Now we extend it to an $I F$-coloring of $G$.

First we color $v_{1}^{\prime}$ and $y$ with the color of the identified vertex. If $v_{1}^{\prime}, y$ are colored $I$, then color $v_{1}, v_{2}, v_{4}$ with $F$ and color $v_{3}$ with a color different from the color of $v_{4}^{\prime}$, and we obtain a valid coloring. So we assume that $v_{1}^{\prime}, y$ are colored $F$. Then there exists no $F$-path between $v_{1}^{\prime}$ and $y$ in $G$. Assume first that $v_{4}^{\prime}$ is colored $I$. If $x$ is colored $I$, then color $v_{1}, v_{3}, v_{4}$ with $F$ and $v_{2}$ with $I$; if $x$ is colored $F$, then color $v_{1}$ with $I$ and $v_{2}, v_{3}, v_{4}$ with $F$. In either case, we obtain a valid coloring. Let $v_{4}^{\prime}$ be colored $F$. If $x$ is colored $I$, then color $v_{2}, v_{4}$ with $I$ and $v_{1}, v_{3}$ with $F$, and we obtain a valid coloring. So we assume $x$ is also colored $F$. Since there is no $F$-path between $v_{1}^{\prime}$ and $y$, either there is no $F$-path between $v_{1}^{\prime}$ and $v_{4}^{\prime}$ or there is no $F$-path between $v_{4}^{\prime}$ and $y$. Also, there is at most one $F$-path between $\left\{v_{1}^{\prime}, y\right\}$ and $C_{0}$. If there is no $F$-path between $v_{1}^{\prime}$ and $C_{0}$ nor between $v_{1}^{\prime}$ and $v_{4}^{\prime}$, then color $v_{1}, v_{4}$ with $I$ and $v_{2}, v_{3}$ with $F$; if there is no $F$-path between $y$ and $C_{0}$ nor between $y$ and $v_{4}^{\prime}$, then color $v_{1}, v_{3}$ with $I$ and $v_{2}, v_{4}$ with $F$; in either of these cases, we obtain a valid coloring. We consider the other two cases.

- Case 1: no $F$-path between $v_{1}^{\prime}$ and $C_{0}$ nor between $y$ and $v_{4}^{\prime}$. We may assume that there are $F$-paths between $v_{1}^{\prime}$ and $v_{4}^{\prime}$ (say $P$ ) and between $y$ and $C_{0}$. Since the $F$-path $P$ in $G$ can be replaced with the $F$-path $y\left(v_{1}^{\prime}\right) P v_{4}^{\prime}$ in $G^{\prime}$, there is no $F$-path between $v_{4}^{\prime}$ and $C_{0}$. Then we can color $v_{1}, v_{3}$ with $I$ and $v_{2}, v_{4}$ with $F$ to obtain a valid coloring.

- Case 2: no $F$-path between $y$ and $C_{0}$ nor between $v_{1}^{\prime}$ and $v_{4}^{\prime}$. We may assume that there are $F$-paths between $v_{1}^{\prime}$ and $C_{0}$ and between $v_{4}^{\prime}$ and $y$ (say $P$ ). Since the $F$-path $P$ in $G$ can be replaced with the $F$-path $v_{1}^{\prime}(y) P v_{4}^{\prime}$ in $G^{\prime}$, there is no $F$-path between $v_{4}^{\prime}$ and $C_{0}$. Then we can color $v_{1}, v_{4}$ with $I$ and $v_{2}, v_{3}$ with $F$ to obtain a valid coloring.

Now suppose that $f$ is incident with five consecutive bad vertices $v_{1}, \ldots, v_{5}$. Since $v_{3}$ is on a 3 -face and $G$ contains no adjacent 3 -faces, either $v_{2} v_{3}$ or $v_{3} v_{4}$ is an edge on a 3 -face. In the former case, $v_{4} v_{5}$ is an edge on a 3 -face; in the latter case, $v_{1} v_{2}$ must be an edge of a 3 -face. This implies that either $v_{2} v_{3} v_{4} v_{5}$ or $v_{1} v_{2} v_{3} v_{4}$ is a tetrad, a contradiction.

For the furthermore part, if a face of $G$ is incident with consecutive vertices $v_{0}, v_{1}, \ldots, v_{5}$ and the vertices $v_{1}, \ldots, v_{4}$ are bad, then either each of $v_{1} v_{2}, v_{3} v_{4}$ is an edge of a 3 -face or each of $v_{0} v_{1}, v_{2} v_{3}, v_{4} v_{5}$ is an edge incident with a triangle. But the former cannot happen, as the path $v_{1} v_{2} v_{3} v_{4}$ is a tetrad and each of $v_{1}, \ldots, v_{4}$ is a bad vertex.

\section{Discharging Procedure}

We are now ready to present a discharging procedure that will complete the proof of Theorem 1.2 , Let $x \in V(G) \cup\left(F(G)-C_{0}\right)$ have an initial charge of $\mu(x)=d(x)-4$, and $\mu\left(C_{0}\right)=\left|C_{0}\right|+4$. By Euler's Formula, $\sum_{x \in V \cup F} \mu(x)=0$. Let $\mu^{*}(x)$ be the charge of $x \in V \cup F$ after the discharge procedure. To lead to a contradiction, we shall prove that $\mu^{*}(x) \geq 0$ for all $x \in V(G) \cup F(G)$ and $\mu^{*}\left(C_{0}\right)>0$.

Let $v$ be a 4 -vertex on a face $f$. The vertex $v \notin C_{0}$ is poor to $f$ if either $v$ is incident with a 3 -face that is not adjacent to $f$, or $v$ is incident with two 3 -faces both adjacent to $f$, or $v$ is incident with a 5 -face adjacent to $f$. A 2-vertex $u \in C_{0}$ is special if $u$ is on an internal 5 -face. 
Here are the discharging rules:

(R1) Each 3 -face gets $\frac{1}{3}$ from each incident vertex.

(R2) Each internal 5 -face gets $\frac{1}{3}$ from each incident $4^{+}$-vertex and gives $\frac{1}{3}$ to each incident 2 -vertex or internal 3 -vertex. Each internal $7^{+}$-face gives $\frac{2}{3}$ to each incident 2 -vertex or bad 3 -vertex, $\frac{1}{3}$ to each incident internal non-bad 3 -vertex or poor 4 -vertex, and then each internal face gives the surplus charge to $C_{0}$.

(R3) Each $4^{+}$-vertex on $C_{0}$ gives $\frac{1}{3}$ to each incident internal 5 -face.

(R4) The outer face $C_{0}$ gives $\frac{5}{3}$ to each incident special 2 -vertex, $\frac{4}{3}$ to each incident non-special 2 -vertex or 3 -vertex on a 3 -face, and 1 to each other incident vertex.

Lemma 3.1. Every vertex $v$ in $G$ has nonnegative final charge.

Proof. We consider the degree of $v$.

Let $d(v)=2$. Then by Lemma $2.1 v \in C_{0}$. By $(\mathrm{R} 2)$ and $(\mathrm{R} 4), v$ gets $\frac{5}{3}$ from $C_{0}$ and $\frac{1}{3}$ from the other incident face when $v$ is special, or gets $\frac{4}{3}$ from $C_{0}$ and $\frac{2}{3}$ from the other incident face otherwise. Thus, $\mu^{*}(v) \geq 2-4+\min \left\{\frac{5}{3}+\frac{1}{3}, \frac{4}{3}+\frac{2}{3}\right\}=0$.

Let $d(v)=3$. If $v \in C_{0}$, then by (R1) and (R4), $v$ gets $\frac{4}{3}$ from $C_{0}$ if $v$ is on a 3-face, or gets 1 from $C_{0}$ otherwise. So $\mu^{*}(v) \geq 3-4+\min \left\{1, \frac{4}{3}-\frac{1}{3}\right\}=0$. Let $v \notin C_{0}$. By (R1) and (R2), $v$ gets $\frac{2}{3}$ from each of the two incident non-triangular faces and gives $\frac{1}{3}$ to its incident 3 -face if $v$ is on a 3 -face, or $v$ gets $\frac{1}{3}$ from each of the three incident faces if $v$ is not bad. So $\mu^{*}(v) \geq 3-4+\min \left\{\frac{1}{3} \cdot 3, \frac{2}{3} \cdot 2-\frac{1}{3}\right\}=0$.

Let $d(v)=4$. Let $v \notin C_{0}$. By (R1) and (R2), $v$ gives $\frac{1}{3}$ to each of (at most two) incident $5^{-}$-faces and gets $\frac{1}{3}$ from each of the two incident $7^{+}$-faces when $v$ is on two 3 -faces; gets $\frac{1}{3}$ from the incident face when $v$ is on exactly one 3 -face and not on a 5-face; gets $\frac{1}{3}$ from each of the two incident $7^{+}$-faces adjacent to the 5 -face when $v$ is on a 5 -face; so $\mu^{*}(v) \geq 4-4+\min \left\{\frac{1}{3} \cdot 2-\frac{1}{3} \cdot 2, \frac{1}{3}-\frac{1}{3}\right\}=0$. When $v \in C_{0}, v$ gets 1 from $C_{0}$ and gives $\frac{1}{3}$ to each of at most three incident faces by (R3), so $\mu^{*}(v) \geq 4-4+1-\frac{1}{3} \cdot 3=0$.

Finally, let $d(v) \geq 5$. If $v \in C_{0}$, then $v$ gets 1 from $C_{0}$ and gives $\frac{1}{3}$ to each of at most $d(v)-1$ incident faces by (R1), (R3) and (R4); if $v \notin C_{0}$, then $v$ gives $\frac{1}{3}$ to each of at most $\left\lfloor\frac{d(v)}{2}\right\rfloor$ incident $5^{-}$-faces by $(\mathrm{R} 1)$ and $(\mathrm{R} 2)$. So $\mu^{*}(v) \geq d(v)-4+\min \left\{1-(d(v)-1) \cdot \frac{1}{3},-\left\lfloor\frac{d(v)}{2}\right\rfloor \cdot \frac{1}{3}\right\}>0$.

Lemma 3.2. Every internal face in $G$ has nonnegative final charge.

Proof. Let $f$ be an internal face in $G$. Recall that $G$ contains no $\{4,6,8\}$-cycles. For $d(f)=3$, $f$ gets $\frac{1}{3}$ from each incident vertex by $(\mathrm{R} 1)$, so $\mu^{*}(f) \geq 3-4+\frac{1}{3} \cdot 3=0$. For $d(f) \geq 5$, by (R2) we only need to show that $f$ has nonnegative charge before it sends charge to $C$, without loss of generality, we still use $\mu^{*}(f)$ to denote it.

Suppose $d(f)=5$. If $V(f) \cap C_{0}=\emptyset$, then $f$ is incident with at most four 3-vertices by Lemma 2.4. By (R2), $f$ gains at least $\frac{1}{3}$ from its incident 4-vertices. If $V(f) \cap C_{0} \neq \emptyset$, then $1 \leq\left|V(f) \cap C_{0}\right| \leq 3$ by Lemma 2.3. If $\left|V(f) \cap C_{0}\right|=1$, then $f$ and $C_{0}$ share a $4^{+}$-vertex, thus by (R3) $f$ gets $\frac{1}{3}$ from the incident vertex on $C_{0}$ and by (R2) gives $\frac{1}{3}$ to each of at most four incident internal 3 -vertices. If $\left|V(f) \cap C_{0}\right|=2$, then $f$ contains no 2-vertices and gives $\frac{1}{3}$ to each of at most three incident internal 3-vertices. If $\left|V(f) \cap C_{0}\right|=3$, then $f$ shares exactly one 2-vertex with $C_{0}$ by Lemma 2.3, thus by (R2), $f$ gives $\frac{1}{3}$ to the 2 -vertex and each of at most two incident internal 3 -vertices. In any case, $\mu^{*}(f) \geq 5-4+\min \left\{-\frac{1}{3} \cdot 4+\frac{1}{3},-\frac{1}{3} \cdot 3\right\}=0$.

Suppose $d(f)=7$. Then $f$ is not adjacent to any 3 -faces and contains at most four 2 -vertices. If $f$ contains a 2-vertex, then $f$ shares at least two $3^{+}$-vertices with $C_{0}$, and $\mathrm{f}$ gives no charge to them. By $(\mathrm{R} 2), \mu^{*}(f) \geq 7-4+\min \left\{-\frac{2}{3} \cdot 4-\frac{1}{3},-\frac{1}{3} \cdot 7\right\}=0$. 
Suppose $d(f) \geq 9$. If $f$ contains 2 -vertices, then $f$ does not give charge to at least two $3^{+}$-vertices shared with $C_{0}$, so by $(\mathrm{R} 2), \mu^{*}(f) \geq d(f)-4-\frac{2}{3}(d(v)-2)>0$. So assume that $f$ contains no 2 -vertices. By Lemma 2.5, $f$ contains at least two vertices that are not bad 3 -vertices. It follows that $\mu^{*}(f) \geq d(f)-4-\frac{2}{3} \cdot(d(f)-2)-\frac{1}{3} \cdot 2 \geq-\frac{1}{3}$, with $\mu^{*}(f)<0$ only if $f$ is a 9 -face with seven bad 3 -vertices and two other internal non-bad 3 -vertices or poor 4 -vertices. But when $f$ contains seven bad 3-vertices, at least four bad 3-vertices are consecutive on $f$. By Lemma 2.5, there are exactly four bad consecutive vertices on $f=v_{1} \ldots v_{9}$, say $v_{1}, v_{2}, v_{3}, v_{4}$, and $v_{9}$ and $v_{5}$ should be $4^{+}$-vertices. As $v_{6}, v_{7}, v_{8}$ are bad 3 -vertices, $v_{5}$ or $v_{9}$, by symmetry say $v_{5}$, is not on another $5^{-}$-face. Then $f$ gives no charge to $v_{5}$ by (R2). Therefore, there is no 9 -face with negative final charge.

Proof of Theorem 1.2. By Lemma 3.1 and Lemma 3.2, it is sufficient for us to check that $C_{0}$ has positive final charge. By Lemma 2.3, if a 5 -face shares 2-vertices with $C_{0}$, then it shares exactly one 2-vertex with $C_{0}$. Let $P$ be a maximal path of $C_{0}$ such that each vertex in $V(P)$ is either a special 2 -vertex or a neighbor of a special 2-vertex. By (R4), $C_{0}$ gives $\frac{5}{3}$ to each incident special 2-vertex and 1 to each neighbor of a special 2-vertex on $C$. So $C_{0}$ gives $\left\lceil\frac{|V(P)|}{2}\right\rceil \cdot 1+\left\lfloor\frac{|V(P)|}{2}\right\rfloor \cdot \frac{5}{3} \leq \frac{4}{3}|V(P)|$ to vertices on $P$. By (R4), $C_{0}$ gives at most $\frac{4}{3}$ to each vertex not in such a path. It follows that $C_{0}$ gives at most $\frac{4}{3}\left|C_{0}\right|$ to its vertices. So $\mu^{*}\left(C_{0}\right) \geq\left|C_{0}\right|+4-\frac{4}{3}\left|C_{0}\right|=\frac{1}{3}\left(12-\left|C_{0}\right|\right) \geq 0$, and $\mu^{*}\left(C_{0}\right)=0$ only when $\left|C_{0}\right|=12$ and either every other vertex on $C_{0}$ is a special 2 -vertex or each vertex on $C_{0}$ is either a 2 -vertex or 3 -vertex on a 3 -face. In the former case, let $u$ be a special 2-vertex on $C_{0}$ and $f^{\prime}$ be the internal 5 -face containing $u$. Let $u_{1}$ and $u_{2}$ be the two neighbors of $u$ on $f^{\prime}$. Since $G$ contains no 8-cycles, $u_{1}$ and $u_{2}$ are $4^{+}$-vertices. By (R2) and (R4), $f$ gets $\frac{1}{3}$ from each of $u_{1}$ and $u_{2}$ and gives at most $\frac{1}{3}$ to each other incident vertex. So $C_{0}$ can get at least $5-4-3 \cdot \frac{1}{3}+\frac{1}{3} \cdot 2=\frac{2}{3}$ from $f^{\prime}$. So $\mu^{*}\left(C_{0}\right)>0$. In the latter case, since $G \neq C_{0}, C_{0}$ contains two bad 3 -vertices that belong to different 3 -faces but on the same face, say $f_{0}$, other than $C_{0}$. It follows that $f_{0}$ is adjacent to at least two 3 -faces, so it must be a $9^{+}$-face since $G$ contains no $\{4,6,8\}$-cycles. By $(\mathrm{R} 2), f_{0}$ gives out at most $\frac{2}{3}\left(d\left(f_{0}\right)-2\right)$ to all incident vertices and gives at least $d\left(f_{0}\right)-4-\frac{2}{3}\left(d\left(f_{0}\right)-2\right) \geq \frac{1}{3}$ to $C_{0}$. So $\mu^{*}\left(C_{0}\right)>0$.

\section{Final Remarks}

Kang, Jin, and Wang [8] proved that planar graphs without $\{4,6,9\}$-cycles are 3 -colorable. We believe that we could get the following result, by slightly modifying the proof of Theorem 1.2 . We will keep it as a future work.

Problem 4.1. Every planar graph without $\{4,6,9\}$-cycles is near-bipartite.

A sketch of proof of Problem 4.1. We use the same discharging rules as the proof of Theorem 1.2. The main difference is that we now have 8-faces to consider but have no 9-faces. In the reducible configurations part, we need to consider the property of the outer face $C_{0}$ carefully since the forbidden cycles have changed. In the discharging part, Now a 7 -face $f$ may share an edge with one 3 -face, but not more. If $f$ contains a 2 -vertex, then it contains at least two $3^{+}$vertices on $C_{0}$, thus gives no charge to them. As $f$ contains at most three 2 -vertices by Lemma 2.3, $\mu^{*}(f) \geq 7-4-3 \cdot \frac{2}{3}-2 \cdot \frac{2}{3}=-\frac{1}{3}$. Then $\mu^{*}(f)<0$ only if $f$ contains three 2 -vertices and one internal triangle with two 3 -vertices on $f$, but we have a separating cycle of length at most 12 in this case. For 8-faces, they do not share edges with triangle and have at most four 2-vertices, so their final charges are at least $8-4-4 \cdot \frac{2}{3}-4 \cdot \frac{1}{3}=0$. The calculation for other vertices and faces is the same as in the proof of Theorem 1.2 . 
We believe that planar graphs without cycles of lengths from 4 to 7 are near-bipartite, but failed to prove it. The trouble is that an 8-face may be adjacent to too many triangles when we try a similar approach to the proof of Theorem 1.2, which we cannot reduce. The condition in Theorem 1.2 excludes 8-cycles, and the conditions in Problem 4.1 excludes the difficult situation.

Acknowledgement: the authors would like to thank Daniel Cranston for bringing their attention to this research problem, and the referees for their careful reading and valuable suggestions.

\section{REFERENCES}

[1] O.V. Borodin, On decomposition of graphs into degenerate subgraphs, Discret Analiz, 28(1976), 3-11.

[2] O. V. Borodin and A. N. Glebov, On the partition of a planar graph of girth 5 into an empty and an acyclic subgraph (Russian), Diskretnyi Analiz i Issledovanie Operatsii, 8(4) (2001), 34-53.

[3] O. V. Borodin, A. N. Glebov, M. Montassier and A. Raspaud, Planar graphs without 5- and 7-cycles and without adjacent triangles are 3-colorable, J. of Combin. Theory Ser. B, 99(2009), 668-673.

[4] O. V. Borodin, A. N. Glebov and A. Raspaud, Planar graphs without triangles adjacent to cycles of length from 4 to 7 are 3-colorable, Discrete Math., 310(2010), 2584-2594.

[5] O. V. Borodin, A. N. Glebov, A. R. Raspaud and M. R. Salavatipour, Planar graphs without cycles of length from 4 to 7 are 3-colorable, J. of Combin. Theory Ser. B, 93(2005), 303-311.

[6] F. Dross, M. Montassier and A. Pinlou, Partitioning a triangle-free planar graph into a forest and a forest of bounded degree, Electronic Notes in Discrete Mathematics, (492)(2015), 269-275.

[7] K. Kawarabayashi and C. Thomassen, Decomposing a planar graph of girth 5 into an independent set and a forest, Journal of Combinatorial Theory, Series B, 99(4)(2009), 674-684.

[8] Y. Kang, L. Jin and Y. Wang, The 3-colorability of planar graphs without cycles of length 4, 6 and 9, Discrete Math., 339 (2016), 299-307.

[9] L. Lovász, Coverings and coloring of hypergraphs, Congressus Numerantium, VIII(1973), 3-12.

[10] C. Thomassen, Decomposing a planar graph into degenerate graphs, J. Combin. Theory Ser. B, 65(1995), 305314.

[11] C. Thomassen, Decomposing a planar graph into an independent set and a 3-degenerate graph, J. Combin. Theory Ser. B, 83 (2001), 262-271.

[12] W. Wang and M. Chen, Planar graphs without 4,6,8-cycles are 3-colorable, Sci. China A , 50 (11) (2007) $1552 \check{\mathrm{s} C}-1562$. 